\title{
Group size and cultural complexity
}

ARISING FRom M. Derex, M.-P. Beugin, B. Godelle \& M. Raymond Nature 503, 389-391 (2013)

A decade ago, Henrich ${ }^{1}$ proposed group size as a driver of cultural complexity. Derex et al. ${ }^{2}$ now present experimental results they say support this 'group size hypothesis' by seemingly showing that larger groups perform better than smaller groups under imitation-based cultural evolution. Our reanalysis of their experimental data, however, shows that larger groups actually perform worse than smaller groups. Thus, contrary to their claim, their data are consistent with empirical evidence discounting the group size hypothesis for non-food producing societies ${ }^{3-10}$. There is a Reply to this Brief Communication Arising by Derex, M. et al. Nature 511, http://dx.doi.org/10.1038/nature13412 (2014).

In their argument, Derex et $a .^{2}$ assume that showing that larger groups produce complex artefacts more often than do smaller groups (see their Fig. 1) suffices for supporting the group size hypothesis. But such an increase can arise just through a sample size effect (for example, we expect to roll more sixes the more dice we roll). Furthermore, the increases in probabilities associated with group size, as shown in their Fig. 1, are even less than what would be expected from the sampling size effect. We believe, therefore, that their data fail to support the group size hypothesis.

We show this statistically by comparing the null hypothesis, $\mathrm{H}_{0}$ : the probability $P$ of an individual drawing a simple artefact on the last step of the experiment is independent of the size of the group to which the individual belongs, with both the one-sided hypothesis that expresses the group size hypothesis, $\mathrm{H}_{1 \mathrm{a}}$ : the probability $p$ of an individual drawing a simple artefact on the last step of the experiment decreases with the size of the group to which the individual belongs, and the alternative one-sided hypothesis that expresses the opposite of the group size hypothesis, namely $\mathrm{H}_{1 \mathrm{~b}}$ : the probability $p$ of an individual drawing a simple artefact on the last step of the experiment increases with the size of the group to which the individual belongs.

We first test whether we can reject $H_{0}$. To do this, we determine the expected performance for each group of size $N=4,8$ or 16 , using the data for groups of size $N=2$ to compute a maximum likelihood estimate $\hat{P}=0.73$ for $P$, the probability that an individual in a group of size $N=2$ draws a simple artefact on the last step of the experiment. We then compute for each group of size $N>2$ the exact probability, using $\hat{P}=0.73$, that the observed performance for a group of size $N$ deviates from the expected performance under $H_{0}$ as much as, or more than, what Derex et al. ${ }^{2}$ observe in their experiments.

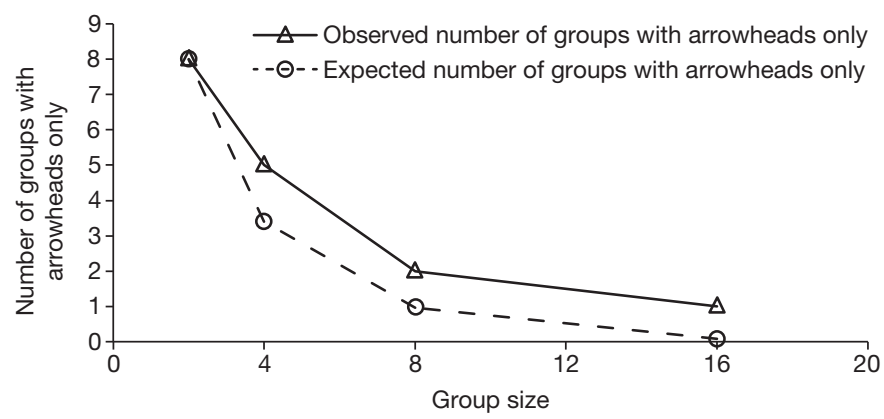

Figure 1 For each group size, observed and expected number of groups drawing only the simple artefact on the last step of the experiment. Expected values are from a maximum likelihood estimate $\hat{P}=0.73$ for the probability $p$ that an individual draws a simple artefact on the last step. The expected number (EN) of groups that only draw simple artefacts is given by $\mathrm{EN}=\hat{P}^{N} \times n$, in which $N$ is the group size and $n$ is the number of groups of size $N(n=15$ for groups of size $N=2$, otherwise $n=12$ ).
The probability $P$ of getting outcomes as extreme as, or more extreme than, the experimental data are given by $P=\operatorname{Pr}\left(X_{4} \geq 5\right) \operatorname{Pr}\left(X_{8} \geq 2\right)$ $\operatorname{Pr}\left(X_{16} \geq 1\right)=(0.235)(0.252)(0.075)=0.0045$ (where $X_{N}$ has a $\operatorname{Bin}(n$, $0.73^{N}$ ) distribution, $N=4,8$ or 16), as (1) there were five groups of size $N=4$, two groups of size $N=8$, and one group of size $N=16$ that only drew the simple artefact on the last step; and (2) the experiments for each group size are mutually independent. As $P<0.005$, we reject $H_{0}$.

Next, we note that the direction of deviation from the expected performance under $H_{0}$ is that of worse performance (see Fig. 1, in which fewer of the groups with $N>2$ have at least one individual drawing a complex artefact on the last step of the experiment than would be expected based on the performance of individuals in groups of size $N=2$ ). Thus, we also reject $H_{1 \mathrm{a}}$ and so we conclude that the data support $H_{1 \mathrm{~b}}$, namely that a larger group size decreases the likelihood of drawing the more complex artefact on the experiment's last step.

In sum, the experimental results of Derex et al. ${ }^{2}$ appear to be contrary to the group size hypothesis. This conclusion also affects negatively their other claims and, in general, underscores the importance of identifying the null hypothesis with care.

\section{Methods}

We compute, for each group size $N>2, \operatorname{Pr}\left(X_{N} \geq x_{N}\right)$, in which $X_{N}$ has a $\operatorname{Bin}(n$, $\left.0.73^{N}\right)$ distribution, $N=4,8$ or $16, n$ is the number of groups of size $N(n=12$ for each of the groups of size $N=4,8$, or 16), and $x_{N}$ is the number of groups of size $N$ that only draw arrowheads on the last step of the experiment.

\section{Claes Andersson ${ }^{1}$ \& Dwight Read ${ }^{2}$}

${ }^{1}$ Physical Resource Theory/Department of Energy and Environment, Chalmers University of Technology, 41296 Göteborg, Sweden.

${ }^{2}$ Department of Anthropology, University of California, Los Angeles, Los Angeles, California 90095, USA.

email: dread@anthro.ucla.edu

\section{Received 15 March; accepted 10 April 2014.}

1. Henrich, J. Demography and cultural evolution: how adaptive cultural processes can produce maladaptive losses: the Tasmanian case. Am. Antiq. 69, 197-214 (2004).

2. Derex, M., Beugin, M.-P., Godelle, B. \& Raymond M. Experimental evidence for the influence of group size on cultural complexity. Nature 503, 389-391 (2014).

3. Collard, M., Kemery, M. \& Banks, S. Causes of toolkit variation among huntergatherers: a test of four competing hypotheses. Can. J. Archaeol. 29, 1-19 (2005)

4. Collard, M., Briggs, B., Ruttle, A. \& O'Brien, M. J. Niche construction and the toolkit of hunter-gatherers and food producers. Biol. Theory 6, 251-259 (2011).

5. Collard, M., Buchanan, B., O'Brien, M. J. \& Scholnick, J. Risk, mobility or population size? Drivers of technological richness among contact-period western North American hunter-gatherers. Phil. Trans. R. Soc. B 368, 20120412 (2013).

6. Collard, M., Buchanan, B. \& O'Brien, M. J. Population size as an explanation for patterns in the Paleolithic archaeological record: more caution is needed. Curr. Anthropol. 54 (S8), S388-S396 (2013).

7. Read, D. Tasmanian knowledge and skill: maladaptive imitation or adequate technology? Am. Antiq. 71, 164-184 (2006).

8. Read, D. An interaction model for resource implement complexity based on risk and number of annual moves. Am. Antiq. 73, 599-625 (2008).

9. Read, D. The misuse of a mathematical model: the Tasmanian case (Reply to Henrich's response). eScholarship Univ. California http://escholarship.org/uc/ item/88k8g4rj (2009).

10. Read, D. Population size does not predict artifact complexity: analysis of data from Tasmania, Arctic hunter-gatherers, and Oceania. eScholarship Univ. California http://www.escholarship.org/uc/item/61n4303q (2012).

Author Contributions D.R. and C.A. contributed equally to this Brief Communications Arising.

Competing Financial Interests Declared none.

doi:10.1038/nature13411 


\section{Derex et al. reply}

REPLYING To C. Andersson \& D. Read Nature 511, http://dx.doi.org/10.1038/nature13411 (2014)

In the accompanying Comment ${ }^{1}$, Andersson \& Read challenge our results $^{2}$ that group size influences cultural complexity. Using a dualtask computer game, our experiment demonstrated that an increasing group size prevents the loss of cultural traits (simple and complex), promotes their improvements and prevents cultural richness to disappear ${ }^{2}$. Among these various effects, Andersson $\& \operatorname{Read}^{1}$ are questioning the finding that larger groups favour the persistence of the complex trait.

Andersson $\&$ Read $^{1}$ claim that the expected number of groups that only exploit the simple task is more than expected if individuals in larger groups were behaving as individuals in smaller groups. Thus, they conclude that group size negatively affects the individual ability to exploit the complex task. Instead our data show the opposite pattern: individuals in larger groups tend to be more able to exploit the complex task (Fig. 1), thus supporting the group size hypothesis.

The first issue with the analysis of Andersson \& $\operatorname{Read}^{1}$ is their estimation of the individual probability to exploit the simple task. Indeed, their method is expected to provide a correct estimation only if individuals behave independently of their other group members. As they claim that individuals' ability is affected by group size, this is quite problematic.

The expected number of groups exploiting only arrowheads should be computed from the probability that a single, isolated individual exploits the simple task. This probability is not available in our data, as individuals were always part of a group. To get around this, we used a generalized linear model on individual data to estimate this probability. We obtained a probability $(P=0.80)$ that a single individual exploits the simple task on the last step of the experiment. Using this probability, we computed the probability of getting outcomes as extreme as, or more extreme than, our observed data for each group size: $\operatorname{Pr}\left(X_{2} \geq 8\right)=0.873$; $\operatorname{Pr}\left(X_{4} \geq 5\right)=0.595 ; \operatorname{Pr}\left(X_{8} \geq 2\right)=0.629$ and $\operatorname{Pr}\left(X_{16} \geq 1\right)=0.295$. Thus, for each group size, the expected number of groups that do not exploit the complex task is not significantly different from expected. We then combine $P$ values using Fisher's method ${ }^{3}$, and obtain an overall $P$ value of 0.79 .

Our initial analysis ${ }^{2}$ showed that the probability of maintaining the complex trait within a group is positively affected by group size. Even if explained by sample size effect, this supports the group size hypothesis: sample size effect is expected to be the main mechanism by which group size affects cultural evolution ${ }^{4-6}$. Sample size effect should promote cultural evolution, unless the individual probabilities to exploit the task decrease drastically with group size. Here our data suggest that the individual probability of exploiting the simple task increases with group size (Fig. 1). In sum, analyses at group and individual levels support the group size hypothesis and are consistent with results from other recent experimental studies ${ }^{7,8}$.

Culture is a group process that arises as a result of underlying individuallevel mechanisms ${ }^{9,10}$. In order to study cultural evolution, two levels of analysis are therefore workable. The analysis of Andersson \& Read ${ }^{1}$ illustrates that individuals' behaviours can hardly be deduced from groups behaviours. Thus, each level of analysis can provide specific information. Depending on the question, analyses should be conducted at one level, or both, but conclusions should always be drawn accordingly.

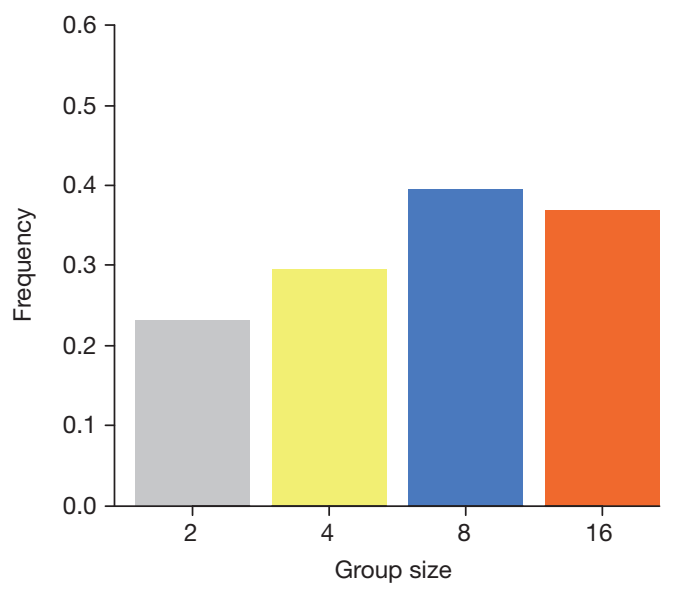

Figure $1 \mid$ Frequency of individuals exploiting the complex task according to group size. Group size had a positive and non-significant effect on the individual probability to exploit the complex task (linear: $\chi^{2}=2.53$, d.f. $=1$, $P=0.11$; quadratic: $\chi^{2}=2.15$, d.f. $=1, P=0.14$ ). Older players tend to be less likely to exploit the complex task $\left(\chi^{2}=3.47\right.$, d.f. $\left.=1, P=0.06\right)$. Players who 'died' during the game ${ }^{2}$ were excluded from the analysis (2-player groups: $n=26$; 4-player groups: $n=44$; 8 -player groups: $n=86$; 16 -player groups: $n=174)$.

\section{Maxime Derex ${ }^{1}$, Marie-Pauline Beugin ${ }^{1}$, Bernard Godelle $^{1}$ \& Michel Raymond ${ }^{\mathbf{1}, 2}$}

${ }^{1}$ University of Montpellier II, Place Eugène Bataillon, 34095 Montpellier Cedex 5, France.

email: maxime.derex@gmail.com

${ }^{2}$ CNRS, Institute of Evolutionary Sciences, CC 065, Place Eugène

Bataillon, Montpellier 34095, France.

1. Andersson, C. \& Read, D. Group size and cultural complexity. Nature 511, http://dx.doi.org/10.1038/nature13411 (2014).

2. Derex, M., Beugin, M.-P., Godelle, B. \& Raymond, M. Experimental evidence for the influence of group size on cultural complexity. Nature 503, 389-391 (2013).

3. Fisher, R. A. Statistical Methods for Research Workers (Oliver and Boyd, 1925).

4. Mesoudi, A. Variable cultural acquisition costs constrain cumulative cultural evolution. PLOS ONE 6, e18239 (2011).

5. Henrich, J. Demography and cultural evolution: how adaptive cultural processes can produce maladaptive losses—the Tasmanian case. Am. Antiq. 69, 197-214 (2004).

6. Shennan, S. Demography and cultural innovation: a model and its implications for the emergence of modern human culture. Camb. Archaeol. J. 11, 5-16 (2001)

7. Muthukrishna, M., Shulman, B. W., Vasilescu, V. \& Henrich, J. Sociality influences cultural complexity. Proc. R. Soc. Lond. B 281, 20132511 (2014).

8. Kempe, M. \& Mesoudi, A. An experimental demonstration of the effect of group size on cultural accumulation. Evol. Hum. Behav. http://dx.doi.org/10.1016/ j.evolhumbehav.2014.02.009 (in the press).

9. Richerson, P. J. \& Boyd, R. Not by Genes Alone (Univ. Chicago Press, 2005).

10. Mesoudi, A. Cultural Evolution: How Darwinian Theory Can Explain Human Culture and Synthesize the Social Sciences (Univ. Chicago Press, 2011).

doi: $10.1038 /$ nature 13412 